\title{
Primary duodenal follicular lymphoma with late disseminated nodal relapse responsive to rituximab monotherapy: A case report
}

\author{
FREDY NEHME $^{1}$, KYLE ROWE $^{1}$, WILLIAM PALKO $^{2}$ and IMAD NASSIF ${ }^{1}$ \\ Departments of ${ }^{1}$ Internal Medicine and ${ }^{2}$ Pathology, Kansas University School of Medicine, Wichita, KS 67214, USA
}

Received May 6, 2017; Accepted August 17, 2017

DOI: $10.3892 /$ mco.2017.1388

\begin{abstract}
Gastrointestinal follicular lymphoma is a rare malignancy accounting for only $1-3.6 \%$ of primary non-Hodgkin lymphomas of the gastrointestinal tract and it is a relatively new clinical entity that was recently classified as a distinct variant of systemic follicular lymphoma. Therefore, data regarding long-term outcome are currently lacking. In addition, a consensus on the management of this disease has not been established and treatment strategies are derived from systemic follicular lymphoma. We herein report the case of a 51-year-old female patient diagnosed with duodenal follicular lymphoma who had nodal relapse nearly 5 years after complete remission. The patient was successfully treated with a 4-week course of rituximab during the initial diagnosis and relapse. To the best of our knowledge, this is the first case report to demonstrate the efficacy of a 4-week course of rituximab during both the initial diagnosis and nodal relapse. The aim of this report was to add to the limited available data on the treatment of gastrointestinal follicular lymphoma.
\end{abstract}

\section{Introduction}

Primary gastrointestinal (GI) follicular lymphoma (FL) is a rare malignancy accounting for only $1-3.6 \%$ of primary non-Hodgkin lymphomas of the GI tract (1). GI-FL is most commonly found within the second portion of the duodenum, but may also involve any part of the small intestine, colon, rectum and stomach $(2,3)$. GI-FL is a relatively new clinical entity and is classified as a distinct variant of systemic FL with a favorable clinical course (4). As several treatment strategies have been proposed, the standard therapy remains to be determined. In the largest case series of GI-FL, rituximab monotherapy was successfully used in 5 patients, resulting in long-term complete remission or stable disease for a median

Correspondence to: Dr Fredy Nehme, Department of Internal Medicine, Kansas University School of Medicine, 1010 N Kansas Street, Wichita, KS 67214, USA

E-mail: nehme.fredy@gmail.com

Key words: follicular lymphoma, rituximab, duodenal cancer, non-Hodgkin lymphoma, gastrointestinal cancer follow-up of 36 months (5). We herein report the case of a 51-year-old female patient with duodenal GI-FL who experienced complete remission after treatment with single-agent rituximab followed by nodal relapse 5 years after treatment completion. The aim of the present case was to add to the limited available data on long-term outcomes of GI-FL and the long-term efficacy of rituximab during initial treatment and relapse.

\section{Case report}

A 51-year-old female patient with a past medical history significant for hypertension presented with history of dyspepsia over several months, despite proton pump inhibitor therapy. The patient reported no fevers, weight loss, decreased appetite, melena or night sweats. Physical examination revealed no abnormalities such as lymphadenopathy, hepatomegaly or splenomegaly. Esophagogastroduodenoscopy (EGD) revealed irregular patchy thickening of the mucosa along the second portion of the duodenum with multiple small white nodular formations (Fig. 1A). Biopsy revealed atypical lymphoid cells with immunostaining positive for CD10, CD20 and B-cell lymphoma 2 (BCL2), consistent with FL. Furthermore, $I g H / B C L 2$ rearrangement was detected on polymerase chain reaction analysis. The diagnosis of GI-FL was confirmed. According to the World Health Organization classification, the pathological grade was 1 (6). Complete staging work-up was performed, including colonoscopy with ileoscopy, computed tomography (CT) scan of the neck, chest, abdomen and pelvis, bone marrow aspirate and biopsy, and positron emission tomography (PET) scan, and the findings were unremarkable. The tumor was stage I according to the Lugano staging classification (7). The Follicular Lymphoma International Prognostic Index 2 (FLIPI2) was 0, corresponding to a 3-year survival rate of $99 \%$ (8). The patient was started on single-agent rituximab $\left(375 \mathrm{mg} / \mathrm{m}^{2} \mathrm{IV}\right.$ per week for a total of 4 weeks). A repeat EGD 3 months after treatment initiation revealed complete resolution of the duodenal lesions (Fig. 1B), and biopsies confirmed normal histology. The patient remained in complete remission on further follow-up studies consisting of yearly endoscopic evaluation of the GI tract and CT scans of the chest, abdomen and pelvis. After 62 months, a follow-up $\mathrm{CT}$ scan of the abdomen reported several mesenteric lymphadenopathies, with the largest lymph nodes measuring 5.4x1.7 and $5.1 \times 2.1 \mathrm{~cm}$ (Fig. 2). A CT of the neck also revealed multiple enlarged cervical lymph nodes bilaterally (Fig. 3). EGD and 

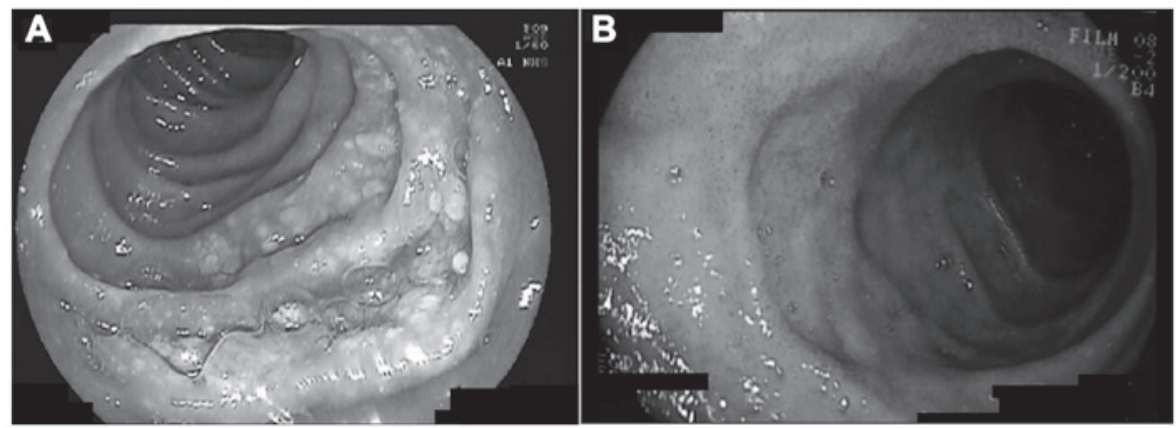

Figure 1. (A) Upper endoscopy revealed multiple white granules in the duodenum. (B) The duodenal lesions disappeared after treatment with rituximab.

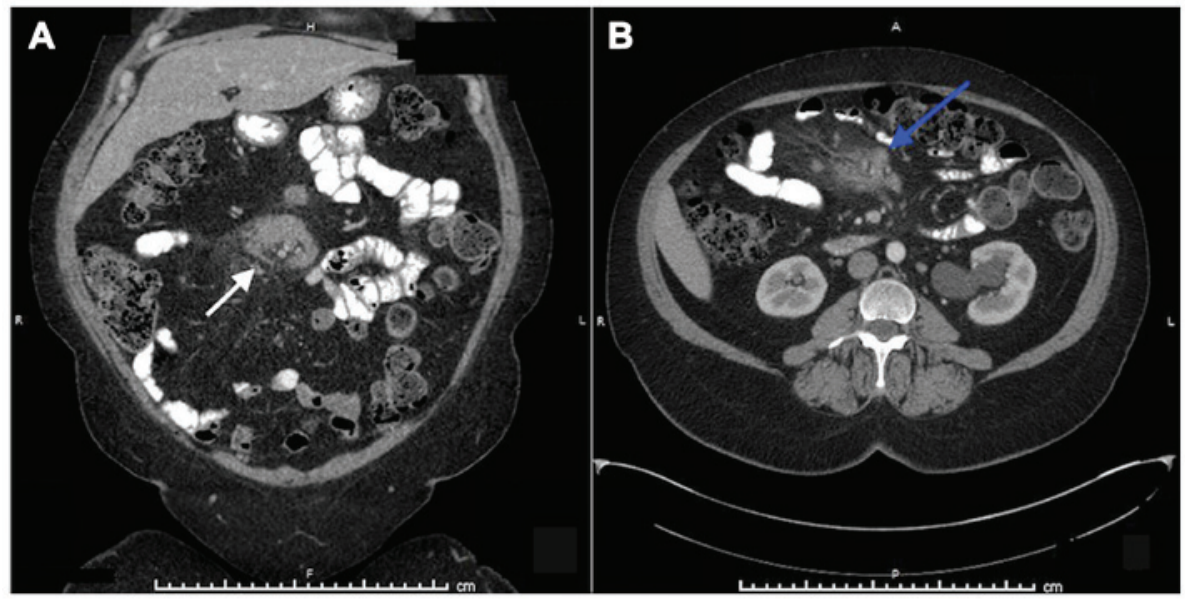

Figure 2. Computed tomography scan of the abdomen showing multiple enlarged mesenteric lymph nodes with the largest lymph nodes measuring (A) $5.1 \times 2.1 \mathrm{~cm}$ (white arrow) and (B) $5.4 \times 1.7 \mathrm{~cm}$ (blue arrow).

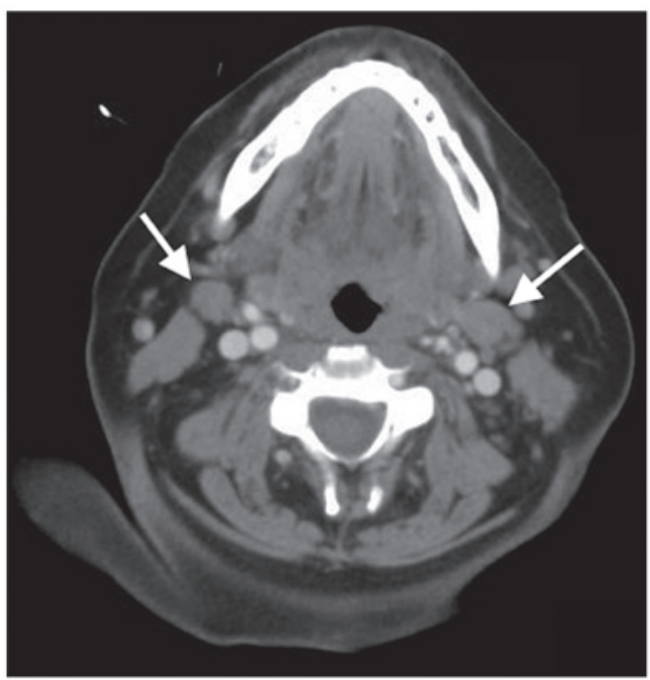

Figure 3. Computed tomography scan of the neck showing multiple enlarged cervical lymph nodes with marker nodes bilaterally at the II B level (arrows).

colonoscopy with biopsies of the duodenal mucosa were normal. PET scanning revealed no additional organ involvement. Biopsy of an enlarged mesenteric lymph node demonstrated recurrent FL (Fig. 4). The patient's FLIPI2 score on recurrence remained 0 , given the absence of bone marrow involvement, normal hemoglobin level, largest involved node sized $<6 \mathrm{~cm}$ and a normal serum $\beta-2$ microglobulin level. The patient was restarted on the same course of rituximab monotherapy as previously described. A follow-up CT scan 2 months after treatment demonstrated complete resolution of the cervical and mesenteric lymphadenopathies. No recurrence has been detected up to 3 years after completion of the second course of rituximab monotherapy (last follow-up, April 2017).

Written informed consent was obtained from the patient for publication of this case report and any accompanying images.

\section{Discussion}

FL, defined as a neoplasm of follicular center B cells, is a frequent indolent lymphoma most commonly of nodal origin (4). Despite the majority of the patients being diagnosed at stage III or IV, the median survival is 8-12 years, leading to the designation of FL as an indolent malignancy (9). Extranodal tissue involvement usually occurs in the setting of disseminated nodal disease. The majority of GI non-Hodgkin lymphomas are either mucosa-associated lymphoid tissue lymphomas or diffuse large B-cell lymphomas (2). GI-FL is a relatively new clinical entity first reported in 1997 (10); since then, it has been reported with increasing frequency and was recently classified as a distinct variant of systemic FL (4). Therefore, data regarding long-term follow-up are scarce. While the majority of patients with nodal FL have systemic disease on presentation, GI-FL rarely disseminates outside of the GI tract, as $93 \%$ of 


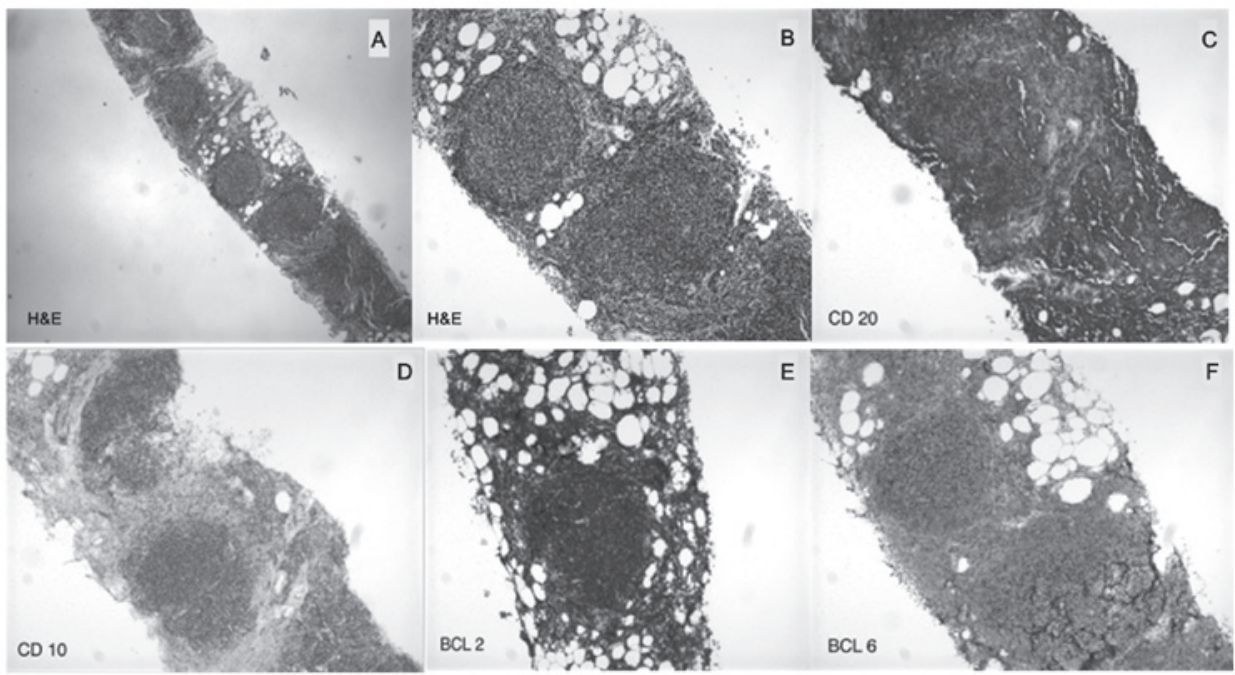

Figure 4. Biopsy revealing infiltration with lymphoid follicular cells (A) hematoxylin and eosin (H\&E) staining, magnification x40 and (B) H\&E staining, magnification x100. Immunohistochemical staining was positive for (C) CD20, (D) CD10, (E) B-cell lymphoma (BCL) 2 and (F) BCL 6.

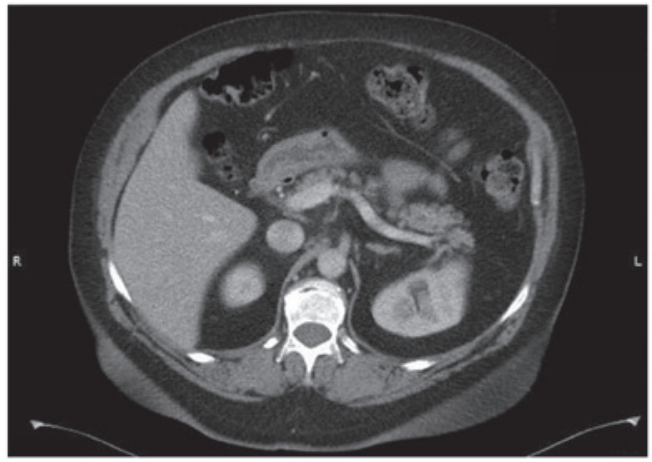

Figure 5. Computed tomography scan of the abdomen showing complete resolution of mesenteric lymphadenopathy.

cases are stage I or II (11). Other distinguishing characteristics include the absence of marginal zone or plasmacytic differentiation and a higher frequency of grade 1 histological grading in GI-FL (11).

Endoscopically, duodenal FL presents with multiple white granules, as shown in the present case (2). Given the potential multifocal involvement of the GI tract, endoscopic evaluation throughout the whole GI tract is recommended. As in nodal $\mathrm{FL}$, the tumor cells are positive for pan B-cell antigens and negative for CD5 and cyclin D1 (12).

Several treatment options for GI-FL have been proposed; however, standard therapy remains to be determined. The currently available options include watchful waiting, radiotherapy, single-agent rituximab, chemotherapy with cyclophosphamide, doxorubicin, vincristine and prednisone (CHOP), rituximab plus CHOP, and radiotherapy plus rituximab (5). However, there are limited data on the effectiveness of each treatment strategy in GI-FL. In general, only 6\% of all patients with GI-FL relapse after initial treatment. It is noteworthy, however, that $50 \%$ of recurrences relapse outside of the GI tract (11). Hence, follow-up evaluation after remission should include whole-body evaluation with PET scan or gallium scintigram. A recent analysis of the Surveillance,
Epidemiology, and End Results database in the United States revealed that small intestinal involvement is an independent predictor of longer survival in GI-FL (13).

Optimal management of GI-FL has not been established. Damaj et al (14) reported no significant difference in the prognosis between patients with and those without treatment. Therefore, the watchful waiting approach has been adopted in several asymptomatic cases $(15,16)$. Given that our patient reported dyspepsia, it was decided to proceed with therapy rather than the watchful waiting approach. After the introduction of rituximab-containing regimens, several randomized controlled trials reported improved complete response rate, duration of the response and overall survival in nodal FL (17-19).

In the largest case series of GI-FL published to date, 5 of 63 patients were treated with rituximab monotherapy. Complete regression occurred in 4 patients, and the fifth patient exhibited stable disease at 118 months of follow-up. However, follow-up was limited to a median of 36 months in this treatment group and the long-term efficacy of rituximab could not be determined (5). A review of the literature by Yamamoto et al in 2010 reported 8 patients treated with rituximab monotherapy as having partial or complete response with no relapse (11). Although prospective studies must be conducted to determine the usefulness of rituximab monotherapy for GI-FL, the previous results indicate that rituximab monotherapy may be an effective therapeutic option. Furthermore, data regarding the optimal dose and duration of rituximab therapy are scarce, as most of the data are extrapolated from standard therapeutic regimens for nodal FL. Several studies demonstrated the long-term efficacy of rituximab monotherapy in nodal FL. Colombat et al reported that $80 \%$ of patients with low tumor burden FL responded to rituximab monotherapy and $48 \%$ achieved complete remission after once-weekly rituximab infusions for a total of 4 doses. Regarding long-term prognosis, the progression-free survival was 23.5 months and the overall survival rate was $91.7 \%$ (20). An analysis of the National LymphoCare Database of stage I FL revealed that diverse treatment approaches, including rituximab monotherapy, radiation therapy, or a combination of radiation therapy and 
chemotherapy, resulted in similar excellent outcomes (21). In general, there appears to be no difference between rituximab and other conventional therapies for GI-FL, possibly due to the favorable prognosis of the disease. In addition, irradiation of the small bowel is associated with chronic radiation enteritis, leading to malabsorption and diarrhea. Therefore, given the potential toxicity of radiation therapy, the effectiveness of rituximab monotherapy, and the indolent nature of GI-FL, single-agent rituximab was selected in this case.

In the present case, single-agent rituximab was used as intravenous infusion once weekly for a total of 4 doses, resulting in complete remission for 62 months. Re-treatment with rituximab was proven to be safe and effective in nodal FL during relapse after initial rituximab exposure (22). Therefore, the same treatment regimen was used during nodal relapse, leading to stable disease up to 3 years after completion of therapy. The efficacy of this regimen in nodal FL was demonstrated in the RESORT trial. In low tumor burden nodal FL, a re-treatment strategy with rituximab $375 \mathrm{mg} / \mathrm{m}^{2}$ for 4 weekly doses upon relapse provides disease control comparable to a rituximab maintenance strategy (23). The present case demonstrated the potential benefit of this protocol in GI-FL during initial treatment and nodal relapse.

In conclusion, despite being considered a rare entity, the reported cases of GI-FL are increasing as is our understanding of its diagnosis, prognosis and treatment. However, a consensus regarding the management of this disease has not been established. GI-FL appears to have a good prognosis, but little is known on the effect of rituximab on the clinical course of GI-FL. The majority of the reported cases responded to rituximab, yet follow-up was limited. The present case demonstrated that rituximab monotherapy is effective in the initial treatment of GI-FL, as well as during systemic relapse. However, further studies are required to determine the optimal treatment regimen.

\section{References}

1. Anderson JR, Armitage JO and Weisenburger DD: Epidemiology of the non-Hodgkin's lymphomas: Distributions of the major subtypes differ by geographic locations. Non-Hodgkin's Lymphoma Classification Project. Ann Oncol 9: 717-720, 1998.

2. Takata K, Okada H, Ohmiya N, Nakamura S, Kitadai Y, Tari A, Akamatsu T, Kawai H, Tanaka S, Araki H, et al: Primary gastrointestinal follicular lymphoma involving the duodenal second portion is a distinct entity: A multicenter, retrospective analysis in Japan. Cancer Sci 102: 1532-1536, 2011.

3. Sentani K, Maeshima AM, Nomoto J, Maruyama D, Kim SW, Watanabe T, Kobayashi Y, Tobinai K and Matsuno Y: Follicular lymphoma of the duodenum: A clinicopathologic analysis of 26 cases. Jpn J Clin Oncol 38: 547-552, 2008.

4. Campo E, Swerdlow SH, Harris NL, Pileri S, Stein H and Jaffe ES: The 2008 WHO classification of lymphoid neoplasms and beyond: Evolving concepts and practical applications. Blood 117: 5019-5032, 2011.

5. Schmatz A-I, Streubel B, Kretschmer-Chott E, Püspök A, Jäger U, Mannhalter C, Tiemann M, Ott G, Fischbach W, Herzog P, et al: Primary follicular lymphoma of the duodenum is a distinct mucosal/submucosal variant of follicular lymphoma: A retrospective study of 63 cases. J Clin Oncol 29: 1445-1451, 2011.

6. Jakić-Razumović J and Aurer I: The World Health Organization classification of lymphomas. Croat Med J 43: 527-534, 2002.

7. Rohatiner A, d'Amore F, Coiffier B, Crowther D, Gospodarowicz M, Isaacson P, Lister TA, Norton A, Salem P, Shipp M, et al: Report on a workshop convened to discuss the pathological and staging classifications of gastrointestinal tract lymphoma. Ann Oncol 5: 397-400, 1994.
8. Federico M, Bellei M, Marcheselli L, Luminari S, Lopez-Guillermo A, Vitolo U, Pro B, Pileri S, Pulsoni A, Soubeyran $\mathrm{P}$, et al: Follicular lymphoma international prognostic index 2: A new prognostic index for follicular lymphoma developed by the international follicular lymphoma prognostic factor project. J Clin Oncol 27: 4555-4562, 2009.

9. Armitage JO and Weisenburger DD: New approach to classifying non-Hodgkin's lymphomas: Clinical features of the major histologic subtypes. Non-Hodgkin's Lymphoma Classification Project. J Clin Oncol 16: 2780-2795, 1998.

10. Misdraji J, Fernandez del Castillo C and Ferry JA: Follicle center lymphoma of the ampulla of Vater presenting with jaundice: Report of a case. Am J Surg Pathol 21: 484-488, 1997.

11. Yamamoto S, Nakase H, Yamashita K, Matsuura M, Takada M, Kawanami $\mathrm{C}$ and Chiba T: Gastrointestinal follicular lymphoma: Review of the literature. J Gastroenterol 45: 370-388, 2010.

12. Bende RJ, Smit LA, Bossenbroek JG, Aarts WM, Spaargaren M, de Leval L, Boeckxstaens GE, Pals ST and van Noesel CJ: Primary follicular lymphoma of the small intestine: alpha4beta7 expression and immunoglobulin configuration suggest an origin from local antigen-experienced B cells. Am J Pathol 162: 105-113, 2003.

13. Chouhan J, Batra S, Gupta R and Guha S: Gastrointestinal follicular lymphoma: Using primary site as a predictor of survival. Cancer Med 5: 2669-2677, 2016.

14. Damaj G, Verkarre V, Delmer A, Solal-Celigny P, Yakoub-Agha I, Cellier C, Maurschhauser F, Bouabdallah R, Leblond V, Lefrère F, et al: Primary follicular lymphoma of the gastrointestinal tract: A study of 25 cases and a literature review. Ann Oncol 14: 623-629, 2003.

15. Kodama M, Kitadai Y, Shishido T, Shimamoto M, Fukumoto A, Masuda H, Tanaka S, Yoshihara M, Sakai A, Nakayama H and Chayama K: Primary follicular lymphoma of the gastrointestinal tract: A retrospective case series. Endoscopy 40: 343-346, 2008.

16. Shia J, Teruya-Feldstein J, Pan D, Hegde A, Klimstra DS, Chaganti RS, Qin J, Portlock CS and Filippa DA: Primary follicular lymphoma of the gastrointestinal tract: A clinical and pathologic study of 26 cases. Am J Surg Pathol 26: 216-224, 2002.

17. Marcus R, Imrie K, Belch A, Cunningham D, Flores E, Catalano J, Solal-Celigny P, Offner F, Walewski J, Raposo J, et al: CVP chemotherapy plus rituximab compared with CVP as first-line treatment for advanced follicular lymphoma. Blood 105: $1417-1423,2005$

18. Hiddemann W, Kneba M, Dreyling M, Schmitz N, Lengfelder E, Schmits R, Reiser M, Metzner B, Harder H, Hegewisch-Becker S, et al: Frontline therapy with rituximab added to the combination of cyclophosphamide, doxorubicin, vincristine and prednisone (CHOP) significantly improves the outcome for patients with advanced-stage follicular lymphoma compared with therapy with CHOP alone: Results of a prospective randomized study of the German Low-Grade Lymphoma Study Group. Blood 106: 3725-3732, 2005

19. Herold M, Haas A, Srock S, Neser S, Al-Ali KH, Neubauer A, Dölken G, Naumann R, Knauf W, Freund M, et al: Rituximab added to first-line mitoxantrone, chlorambucil, and prednisolone chemotherapy followed by interferon maintenance prolongs survival in patients with advanced follicular lymphoma: An east german study group hematology and oncology study. J Clin Oncol 25: 1986-1992, 2007.

20. Colombat P, Brousse N, Salles G, Morschhauser F, Brice P, Soubeyran P, Delwail V, Deconinck E, Haioun C, Foussard C, et al: Rituximab induction immunotherapy for first-line low-tumor-burden follicular lymphoma: Survival analyses with 7-year follow-up. Ann Oncol 23: 2380-2385, 2012.

21. Friedberg JW, Byrtek M, Link BK, Flowers C, Taylor M, Hainsworth J, Cerhan JR, Zelenetz AD, Hirata J and Miller TP: Effectiveness of first-line management strategies for stage I follicular lymphoma: Analysis of the National LymphoCare Study. J Clin Oncol 30: 3368-3375, 2012.

22. Davis TA, Grillo-López AJ, White CA, McLaughlin P, Czuczman MS, Link BK, Maloney DG, Weaver RL, Rosenberg J and Levy R: Rituximab anti-CD20 monoclonal antibody therapy in non-Hodgkin's lymphoma: Safety and efficacy of re-treatment. J Clin Oncol 18: 3135-3143, 2000.

23. Kahl BS, Hong F, Williams ME, Gascoyne RD, Wagner LI, Krauss JC, Habermann TM, Swinnen LJ, Schuster SJ, Peterson CG, et al: Rituximab extended schedule or re-treatment trial for low-tumor burden follicular lymphoma: Eastern cooperative oncology group protocol e4402. J Clin Oncol 32: 3096-3102, 2014. 